\title{
16. Mainzer Allergie-Workshop 2004
}

\section{Neue Tripeltherapie bei chronischer Urtikaria}

\author{
Die Behandlung der chronischen Urtikaria stellt für den Allergo- \\ logen immer wieder eine Herausforderung dar. Zwar ist die gängige \\ Therapie mit H1-Antagonisten oft ausreichend, sie führt jedoch - \\ auch in sehr hohen Dosierungen - nicht immer zum gewünschten \\ Erfolg.
}

E ine Arbeitsgruppe der Ludwig-Mastellte in Mainz einen interessanten neuen Ansatz zur Behandlung therapierefraktärer Urtikaria vor. Präzedenzfall war eine 40-jährige Patientin, die seit etwa einem Jahr unter chronischer Urtikaria litt. Bei der Fokussuche waren erhöhte Mykoplasmen-Antikörpertiter und Candida im Stuhl aufgefallen, woraufhin eine Therapie mit Doxycyclin und Amphotericin B über 4 Wochen durchgeführt wurde. Doch sowohl dieser kausale Therapieansatz als auch die symptomatische Therapie mit Fexofenadin, Levocetirizin und Chloroquin erbrachten keine befriedigenden Ergebnisse.

\section{Erfolg mit Dreier- kombination}

Eine nun begonnene Tripeltherapie, bestehend aus dem Antihistaminikum Levocetirizin $(5 \mathrm{mg} / \mathrm{d})$, dem Leukotrienantagonisten Montelukast $(10 \mathrm{mg} / \mathrm{d})$ und Rofecoxib $(25 \mathrm{mg} / \mathrm{d})$, einem selektiven Inhibitor der Cyclooxygenase 2 (COX 2), führte innerhalb von kurzer Zeit zu einer deutlichen Besserung der Symptomatik. Nach etwa 4-wöchiger Behandlungsdauer wurde die Notwendigkeit der einzelnen Komponenten überprüft: Das Absetzen sowohl von Rofecoxib oder auch von Montelukast hatte eine sofortige Ver- schlechterung zu Folge, woraufhin die Dreiertherapie erneut und mit anhaltendem Erfolg fortgeführt wurde.

\section{Einzelfall oder allgemein gültig?}

Die viel versprechende neue Tripeltherapie wurde mittlerweile an neun weiteren Patienten im Alter zwischen 20 und 58 Jahren überprüft. Sie litten seit 2-130 Monaten unter Urtikaria, die bisher mit Antihistaminika nur unbe-

friedigend beherrscht werden konnte. Tatsächlich kam es bei acht der neun Personen unter der Tripeltherapie zu weitgehender oder sogar vollständiger Erscheinungsfreiheit. Bei einer Patientin blieben die Symptome unverändert. Die Wirkung trat meist verzögert, nach 5-14 Tagen, ein. Bei zwei Patienten traten auch nach dem Absetzen der Therapie keine weiteren Quaddelschübe auf.

Bisher war eine Wirkung der einzelnen Komponenten nur bei alleiniger Gabe oder in Zweierkombinationen in Studien erprobt worden. So hatte der Leukotrienantagonist Zafirlukast in einer Studie keine Überlegenheit gegenüber Plazebo gezeigt, während mit einer Kombination aus Antihistaminika plus COX-2-Hemmer bereits zuvor Erfolge in der Behandlung der chronischen Urtikaria erzielt worden waren. Der Therapieansatz der Dreierkombination scheint jedoch angesichts der pathophysiologischen Grundlagen der Entstehung von Quaddeln äußerst plausibel. Hier spielen - wie in der Grafik gezeigt - Histamin, Cyclooxygenase 2 und Leukotriene eine Schlüsselrolle.

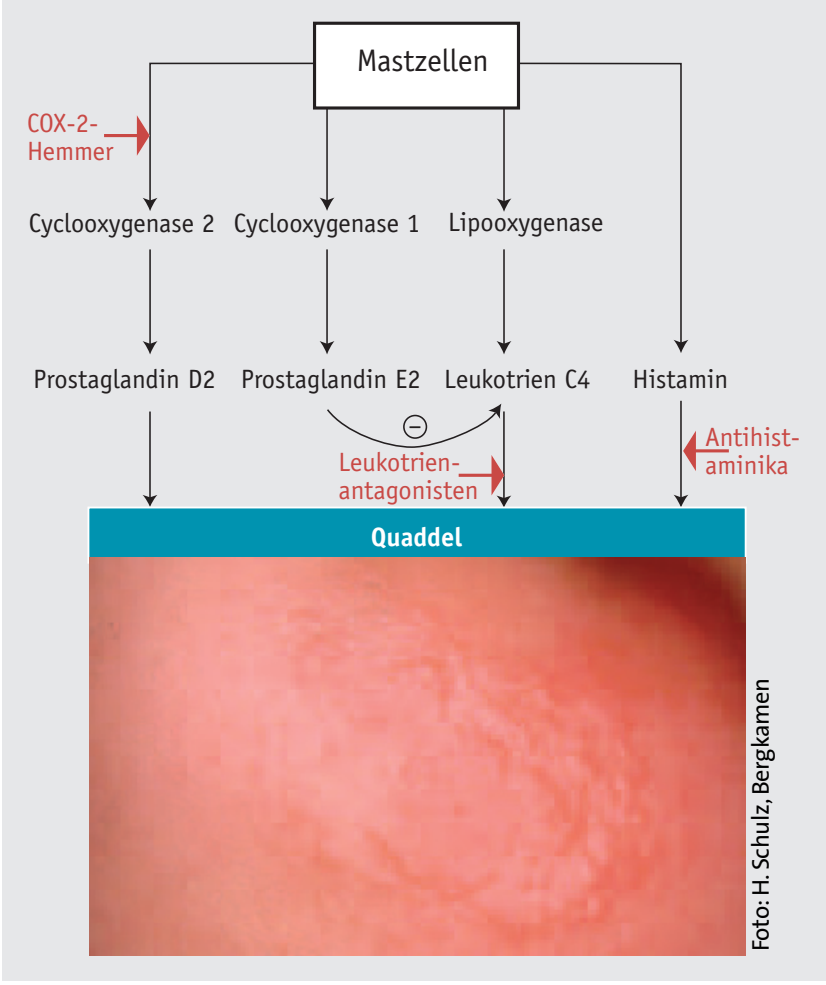

Eine Tripeltherapie aus COX-2-Inhibitor, Leukotrienantagonist und Antihistaminikum durchbricht drei verschiedene Reaktionskaskaden, die von der degranulierenden Mastzelle zur Quaddel führen.

\section{Weitere Studien nötig}

Die Ergebnisse der bisherigen Untersuchungen zeigen, dass eine Tripeltherapie aus Antihistaminikum, Leukotrienrezeptor-Antagonist und selektivem COX-2-Hemmer bei therapierefraktärer chronischer Urtikaria erfolgreich sein kann. Welchen Stellenwert diese Therapiekombination in Zukunft in der Behandlung der chronischen Urtikaria einnehmen wird, muss jedoch erst noch in kontrollierten Studien untersucht werden.

Oppel T et al. Montelukast und Rofecoxib in Kombination mit Antihistaminikum zur Behandlung der therapierefraktären chronischen Urtikaria. 16. Mainzer AllergieWorkshop, Mainz, 12.-13. März 2004 